glaucoma. It is now recognised to comprise the diagnostic triad of a diffusely flat anterior chamber, high intraocular pressure, and aqueous pooling that is sometimes visible in or in front of the anterior vitreous.

Malignant glaucoma is notoriously difficult to treat and carries a generally poor prognosis for long term control of intraocular pressure.

It is thought to involve the mechanisms of ciliolenticular block of aqueous flow leading to the misdirection of aqueous posteriorly into, or in front of, the vitreous gel leading to the characteristic diffuse shallowing of the anterior chamber accompanied by an often precipitous rise in intraocular pressure. Medical treatment alone is rarely successful in establishing control of the IOP. ${ }^{23}$ Pars plana vitrectomy has been used in the surgical management of malignant glaucoma with some definite but limited success in phakic as well as pseudophakic eyes. ${ }^{45}$

However, when malignant glaucoma develops in pseudophakic eyes (with posterior chamber IOLs) there exists an additional treatment option of Nd:YAG laser capsulotomy and/or vitreolysis $^{26}$ which can be effective in arresting the spiralling rise in IOP by redirecting aqueous flow anteriorly thereby avoiding the need for acute surgical intervention with its inevitably higher morbidity. The same reasoning can be applied to aphakic eyes that have an intact posterior capsule, with similar chances of a successful outcome.

1 von Graefe A. Bietrage zur pathologie und therapie des glaucoms. Arch Ophthalmol 1869; 15: 108

2 Tomey KF, Senft SH, Antonios SR, Shammas IV, Shihab SM, Traverso CE. Aqueous misdirection and flat chamber after Traverso CE. Aqueous misdirection and flat chamber after tomy. Arch Ophthalmol 1987; 105: 770-3.

3 Schrader CE, Belcher CD III, Thomas JV, Simmons RJ, Murphy EB. Pupillary and iridovitreal block in pseudophakic eyes. Ophthalmology 1984; 91: 831-7.

4 Weiss H, Shin DH, Kollarits CR. Vitrectomy for malignant (ciliary block) glaucomas. Int Ophthalmol Clin 1981; 21: 113.

5 Lynch MG, Brown RH, Michels RG, Pollack IP, Stark WJ. Surgical vitrectomy for pseudophakic malignant glaucoma. Aurgical vitrectomy for pseudophak

6 Little BC, Hitchings RA. Pseudophakic malignant glaucoma: Nd:YAG capsulotomy as a primary treatment. Eye 1993; 7: $102-4$.

\title{
Nasolacrimal obstruction and facial bone histopathology in craniodiaphyseal dysplasia
}

\author{
Dominic A McHugh, Geoffrey E Rose, Alec Garner
}

Moorfields Eye Hospital, City Road, London EC1V 2PD

D A McHugh

G E Rose

A Garner

Correspondence to:

Mr G E Rose.

Accepted for publication

10 January 1994
Craniodiaphyseal dysplasia is a rare, severe, and progressive bone dysplasia characterised by thickening, distortion, and enlargement of the cranium and face. ${ }^{12}$ Epiphora and recurrent dacrycystitis can occur in this and other such bone diseases owing to nasolacrimal duct stenosis. ${ }^{34}$ The management of such a case provided an opportunity to obtain material for analysis, with the aim of gaining more information on the aetiology and possible treatment of this condition.

\section{Case report}

The patient was a female, aged 3 years in whom a diagnosis of craniodiaphyseal dysplasia had been

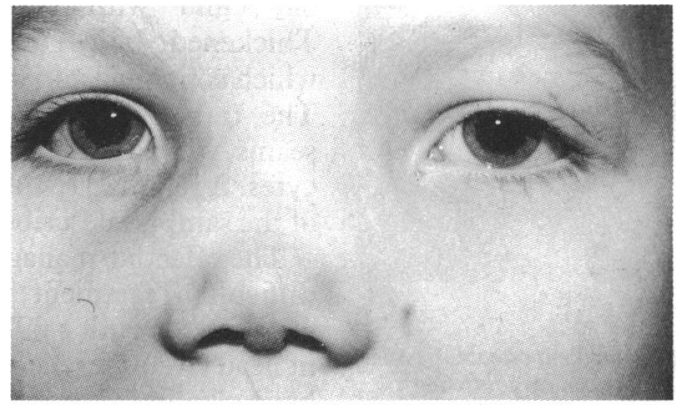

Fig $1 A$ made soon after birth, from the typical clinical (Fig 1A) and radiological appearances (Fig 1B). $A$ low calcium diet had been commenced and calcitriol therapy administered in an attempt to

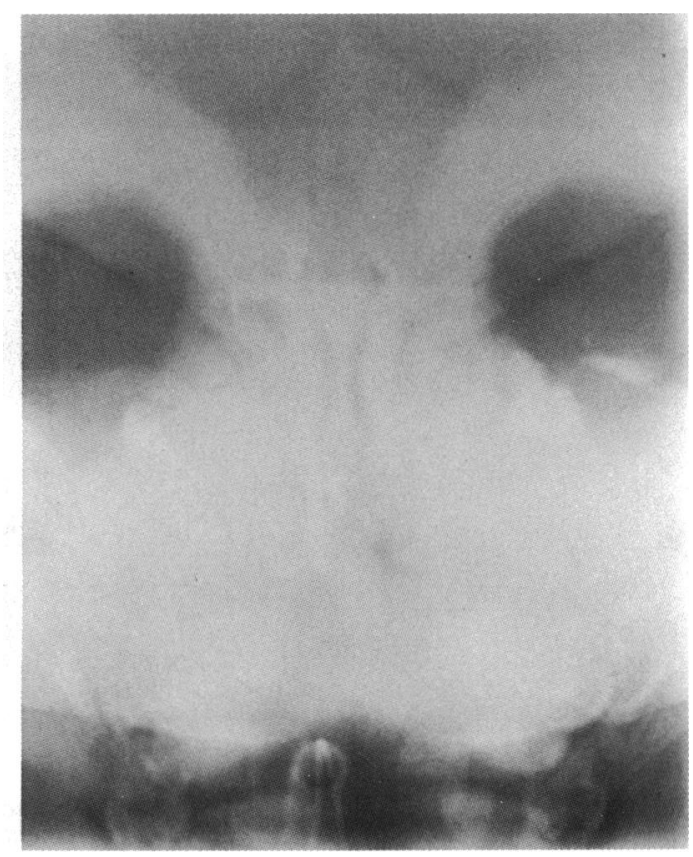




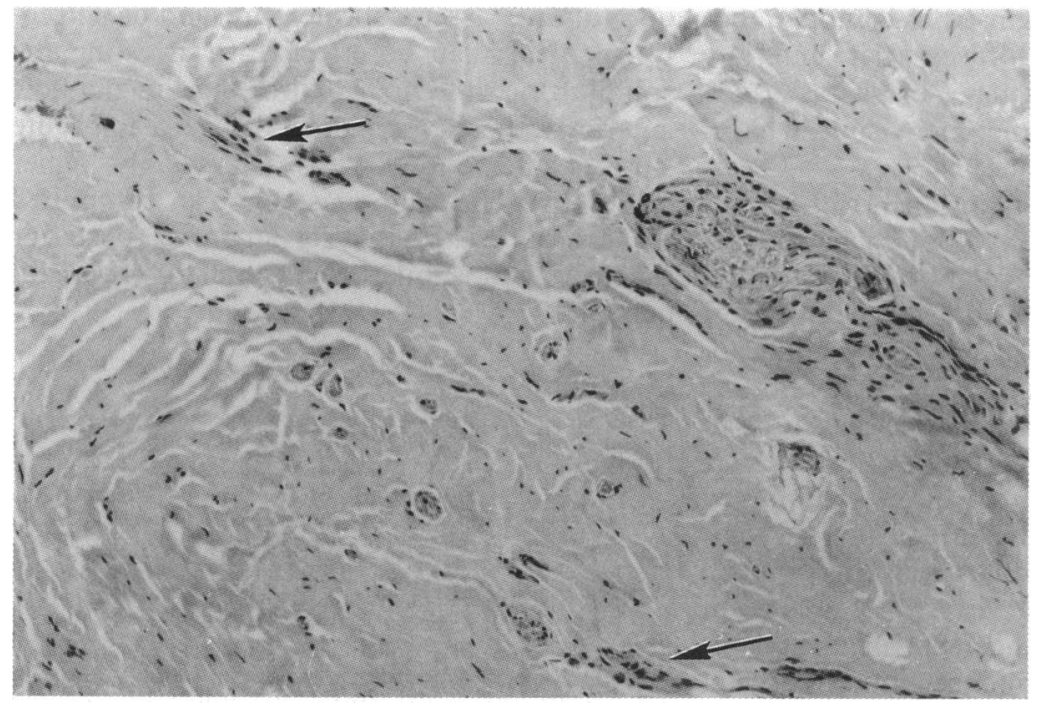

Figure 2 Histology of the bony fragments obtained during surgery disclosed a thickened periosteum composed of dense, hyalinised fibrous tissue with focal clusters of fibrocytes (arrows). A nerve twig is also seen. (Haematoxylin and eosin, $\times 115$ ).

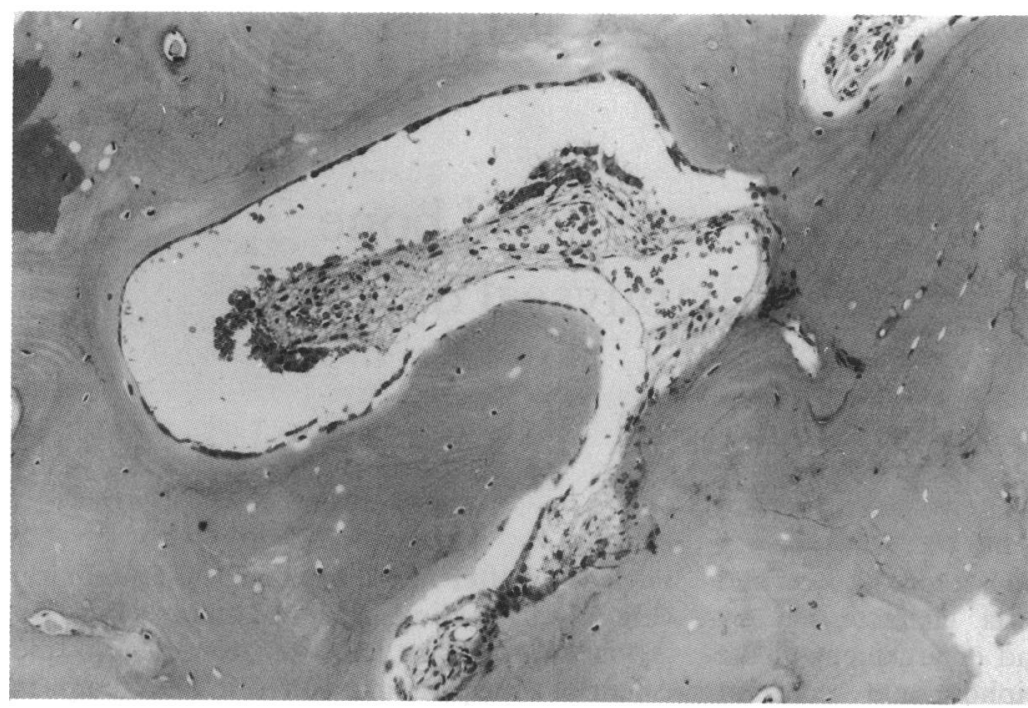

Fig $3 A$

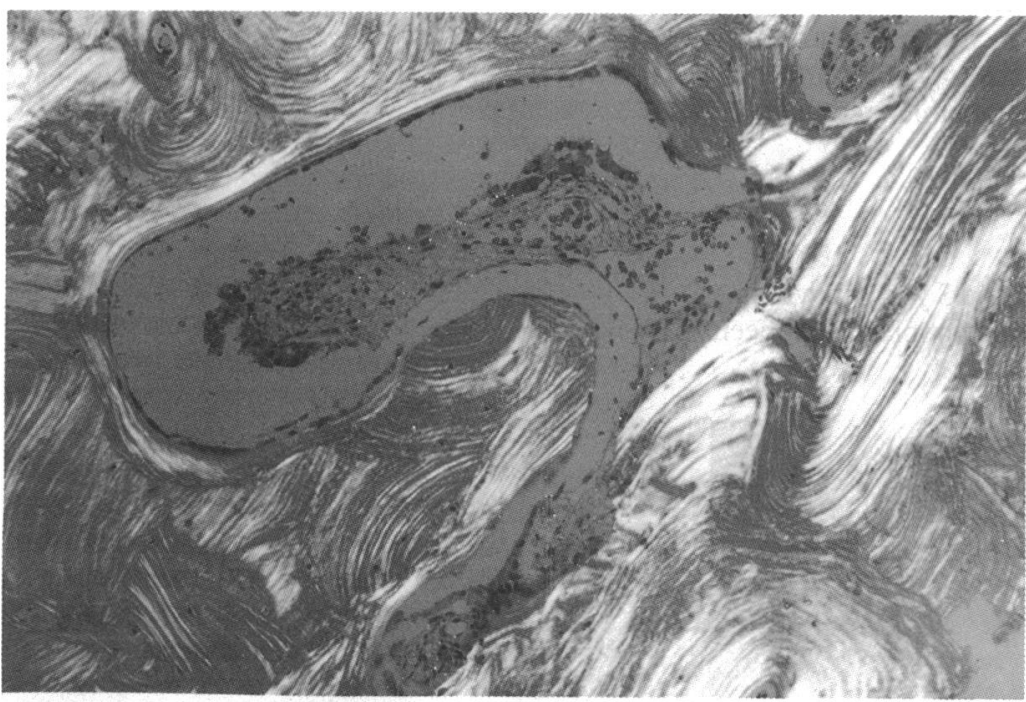

Fig $3 B$

Figure 3 Cortical lamellar bone. (A) A remodelled space lined by prominent osteoblasts and containing fibrous tissue infiltrated with lymphocytes. (B) Same field viewed by polarising microscopy emphasises the remodelled nature of the lamellar bone. (Haematoxylin and eosin,
$\times 115$ ). increase calcium excretion and thus promote bone absorption. Despite treatment, there was progression of the condition and she developed left sided nasolacrimal duct obstruction with epiphora and recurrent dacryocystitis. At the age of 2, a dacryocystorhinostomy (DCR) was performed at the referring hospital, but her symptoms recurred 9 months later.

A second DCR, with insertion of O'Donoghue silicone tubes was performed. A region of new bone formation, approximately $2 \mathrm{~cm}$ thick was seen, this appearing to almost completely occlude the previous anastomosis. A large $(2 \times 1 \mathrm{~cm})$ rhinostomy was fashioned and a $2 \mathrm{~cm}$ nasal mucosal flap was sutured to the anterior leaf of the lacrimal sac. Bone fragments were processed for light microscopy and revealed a periosteum composed of dense, hyalinised fibrous tissue containing focal cell clusters of fibrocytes and lymphocytes (Fig 2). Cortical lamellar bone showed evidence of new bone formation, with remodelling and osteoid seams lined by unusually prominent osteoblasts (Fig 3). Six months after surgery, the silicone tubes were removed under general anaesthesia and after 18 months, there has been no recurrence of her lacrimal symptoms.

\section{Comment}

There are fewer than 20 reported cases of craniodiaphyseal dysplasia. A recessive mode of inheritance has been suggested. ${ }^{5}$ The underlying pathology appears to be hyperostosis and sclerosis, predominantly affecting the skull and facial bones. ${ }^{12}$ Other skeletal abnormalities include a cylindrical appearance of the long bones and thickening and sclerosis of the ribs, clavicles, pelvis, and spine. ${ }^{6}$ Growth may be retarded and sexual maturation delayed. An early death is common. ${ }^{2}$

Bony overgrowth can result in cranial nerve compression, causing visual and auditory impairment and may be associated with headache, mental retardation, or seizures. ${ }^{7}$ Craniofacial abnormalities include paranasal bossing and hypertelorism (Fig 1A), which may cause lacrimal or nasal obstruction, resulting in recurrent lacrimal and respiratory tract infections. ${ }^{8}$

Although the bony abnormalities are usually confirmed by radiological examination (Fig 1B), in this case we were able to provide a histological indication of the underlying pathology (Figs 2 and 3). Although relatively non-specific, they are similar to the morphological findings of Bonucci and colleagues who examined biopsy samples from the frontal bone and iliac rest of a 5-yearold child with craniodiaphyseal dysplasia. ${ }^{9}$ Thickened bony trabeculae were observed, which contained variable numbers of osteocytes. The trabeculae were in contact with osteoid seams which contained osteoblasts and osteocytes. A relative lack of calcification was a feature of the samples described.

The effective management of this condition is difficult. Treatment with calcitonin has been reported to result a fall in the velocity of increase in head circumference and changes in serum and bone alkaline phosphatase ${ }^{10}$ Compression of the cranial foramina may result in brain stem com- 
pression owing to cerebral oedema; the foramina typically close later, because of further bony overgrowth. ${ }^{3}$ In allied conditions such as osteopetrosis, lacrimal surgery may be successful initially, but ossification of the rhinostomy may require further surgery. ${ }^{4}$ It is hoped that in the present case, modification of the disease process by medical therapy and the fashioning of a large rhinostomy might provide a longer lasting palliation of the symptoms of this rare, but distressing condition.

1 Macpherson RI. Craniodiaphyseal dysplasia, a disease or a group of diseases? f Canad Assoc Radiol 1974; 25: 22-33.

2 Brueton LA, Winter RM. Craniodiaphyseal dysplasia. 7 Med Genet 1990; 27 ; 701-6.
3 Beighton P, Hamersma H. Ophthalmological complications in the sclerosing bone dysplasia. Ophthalmol Paedr Gen 1985; 6: 129-34.

4 Orengo SD, Patrinely JR. Dacryocystorhinostomy in osteopetrosis. Ophthalmic Surg 1991; 22: 396-8.

5 de Souza O. Leontiosis ossea case reports. Porto Allegre (Brazil) Fac Med 1972; 13: 46-54.

6 Tucker AS, Klein L, Antony GJ. Craniodiaphyseal dysplasia: evolution over a 5 year period. Skeletal Radiol 1976; 1: 47-53.

7 Stransky E, Mabilangan L, Lara RT. On Paget's disease with leontiasis ossea and hypothyreosis, starting in early childhood. Ann Paediatr (Paris) 1962; 199: 393-408.

8 Halliday J. A rare case of bone dystrophy. Brf Surg 1949; 37: 52-63.

9 Bonucci E, Menichini G, Scarfo GB, Tomaccini D. Histologic, microradiographic and electron microscopic investigations of bone tissue in a case of craniodiaphyseal dysplasia. Virchows Arch A Anat Histol 1977; 373: 167-75.

10 McKeating JB, Kershaw CR. Craniodiaphyseal dysplasia. Partial suppression of osteoblastic activity in the severe progressive form with calcitonin therapy. $f R$ Nav Med 1987; 73: 81-93.

\title{
An unusual form of blink reflex induced by pressing on a frontal burr hole
}

\author{
Avinoam B Safran, Gian Luca Laffi, Bertrand Demierre
}

Department of

Ophthalmology, Geneva University Hospital, Geneva, Switzerland

Neuro-ophthalmology Unit

A B Safran

G L Laffi

Department of Neurosurgery B Demierre

*Current address: Department of Ophthalmology, Ottica Fisiopatologica (Pf Meduri), Bologna University Hospital, Bologna Italy.

Correspondence to: Professor Avinoam B Safran, Neuro-ophthalmology Unit, Geneva University Hospital 1211 Geneva 14, Switzerland. Accepted for publication 10 January 1994
In a patient with Arnold-Chiari type I malformation and drained hydrocephalus communicans, we observed the unique clinical pattern of a sustained contraction in the orbicularis oculi muscle when pressing on the burr hole. It can be speculated that digital pressure on the dura mater elicited an unusual form of blink reflex. It is also conceivable that this phenomenon was related to a slight increase in intracranial pressure.

\section{Case report}

A 60-year-old woman complained of intermittent contractions of the right orbicularis oculi muscle. She presented an Arnold-Chiari type I malformation, with closed Magendie foramen. She had successively undergone a ventriculoperitoneal shunt, numerous external ventricular drainages through a right frontal burr hole, suboccipital craniectomy with C1-C2 laminectomy, and opening of the Magendie foramen. Three years later, she noted narrowing of the right palpebral fissure when pressing with her finger on the scalp overlying the frontal burr hole. She also complained of vertical diplopia and difficulty in walking.

Neuro-ophthalmic examination showed that visual acuity and visual fields were normal. The patient showed signs of left ocular tilt reaction with tilting of the head to the left, left comitant hypotropia 6 dioptres in magnitude, and conjugate leftward cyclotorsion. Corneal sensitivity and resting position of the eyelids were unremarkable. However, when pressing with the finger on the scalp overlying the bone aperture previously made by trephination, a sustained contraction of the orbicularis oculi occurred.
Only the pretarsal fibres of the lower part of the orbicularis muscle were involved. This phenomenon was observed each time, and as long as, digital pressure was applied in the trephination area. Neurological examination further showed slight ataxia and instability without cerebellar syndrome.

\section{Comment}

This patient showed a most unusual phenomenon, consisting of a unilateral contraction of orbicularis oculi muscle which occurred when pressing on a burr hole in the frontal bone. The mechanisms which produce this phenomenon are poorly understood. The possibility of a voluntary contraction of the eyelid was discarded because of the sectorial involvement of fibres in the orbicularis oculi muscle. We suggest that the pressure applied on the frontal burr hole irritated an area of the dura mater innervated by the trigeminal nerve, ${ }^{1}$ thereby inducing an unusual, segmental form of blink reflex.

A second hypothesis, although much less probable, may be considered to explain the occurrence of the lid phenomenon reported here. It is based on a possible increase in intracranial pressure caused by pressing on the burr hole. Indeed, it cannot be excluded that the blinking was a sign of increased intracranial pressure, causing irritation of the facial nerve, even though the rise in intracranial pressure induced by digital pressure was probably moderate. Cranial nerve function is occasionally altered following elevation of intracranial pressure. The most common changes involve the optic and the abducens nerves. ${ }^{2}$ In this condition, single cases 